\title{
LAGUERRE-GAUSS FILTERS IN REVERSE TIME MIGRATION IMAGE RECONSTRUCTION
}

\author{
Juan Guillermo Paniagua Castrillón ${ }^{1}$, Olga Lucia Quintero Montoya² and Daniel Sierra-Sosa ${ }^{3}$
}

\begin{abstract}
Reverse time migration (RTM) solves the acoustic or elastic wave equation by means of the extrapolation from source and receiver wavefield in time. A migrated image is obtained by applying a criteria known as imaging condition. The cross-correlation between source and receiver wavefields is the commonly used imaging condition. However, this imaging condition produces spatial low-frequency noise, called artifacts, due to the unwanted correlation of the diving, head and backscattered waves. Several techniques have been proposed to reduce the artifacts occurrence. Derivative operators as Laplacian are the most frequently used. In this work, we propose a technique based on a spiral phase filter ranging from 0 to $2 \pi$, and a toroidal amplitude bandpass filter, known as Laguerre-Gauss transform. Through numerical experiments we present the application of this particular filter on three synthetic datasets. In addition, we present a comparative spectral study of images obtained by the zero-lag cross-correlation imaging condition, the Laplacian filtering and the Laguerre-Gauss filtering, showing their frequency features. We also present evidences not only with simulated noisy velocity fields but also by comparison with the model velocity field gradients that this method improves the RTM images by reducing the artifacts and notably enhance the reflective events.
\end{abstract}

Keywords: Laguerre-Gauss transform, zero-lag cross-correlation, seismic migration, imaging condition.

RESUMO. A migração reversa no tempo (RTM) resolve a equação de onda acústica ou elástica por meio da extrapolação a partir do campo de onda da fonte e do receptor no tempo. Uma imagem migrada é obtida aplicando um critério conhecido como condição de imagem. A correlação cruzada entre campos de onda de fonte e receptor é a condição de imagem comumente usada. No entanto, esta condição de imagem produz ruído espacial de baixa frequência, chamados artefatos, devido à correlação indesejada das ondas de mergulho, cabeça e retrodifusão. Várias técnicas têm sido propostas para reduzir a ocorrência de artefatos. Operadores derivados como Laplaciano são os mais utilizados. Neste trabalho, propomos uma técnica baseada em um filtro de fase espiral que varia de 0 a $2 \pi$, e um filtro passabanda de amplitude toroidal, conhecido como transformada de Laguerre-Gauss. Através de experimentos numéricos, apresentamos a aplicação deste filtro particular em três conjuntos de dados sintéticos. Além disso, apresentamos um estudo comparativo espectral de imagens obtidas pela condição de imagem de correlação cruzada atraso zero, a filtragem de Laplaciano e a filtragem Laguerre-Gauss, mostrando suas características de frequência. Apresentamos evidências não somente com campos simulados de velocidade ruidosa, mas também por comparação com os gradientes de campo de velocidade do modelo que este método melhora as imagens RTM, reduzindo os artefatos e aumentando notavelmente os eventos reflexivos.

Palavras-chave: transformação de Laguerre-Gauss, correlação cruzada atraso zero, migração sísmica, condição de imagem.

\footnotetext{
1 Instituto Tecnológico Metropolitano - ITM, Faculty of Engineering, Calle 73 N 76A 354, Bloque G-103, Medellín, Colombia. Phone: (57 4) 440-5100 Ext 5161 -E-mails: juanpaniagua@itm.edu.co / jgpaniagu@eafit.edu.co

2Universidad EAFIT, Department of Mathematical Sciences, Carrera 49 N 7 sur, 50, Bloque 38-434, Medellín, Colombia. Phone: (57 4) 261-9500, Ext 9064 -E-mail: oquinte1@eafit.edu.co

3 University of Louisville, Computer Engineering and Computer Science Department, Duthie Center for Engineering, Louisville, KY 40292, USA, 222 Eastern Parkway, Louisville, Kentucky 40209 - E-mail: dsierrasosa@gmail.com
} 


\section{INTRODUCTION}

Reverse time migration (RTM) solves the two-way acoustic or elastic wave equation, by the propagation in time domain of the source wavefield in forward direction, and of the receiver wavefield in backward direction. The migrated image is obtained by the zero-lag cross-correlation (ZL-CC) by examining the double summation of products of seismic amplitudes between the source and the receiver wavefields. One of them, summed in time domain and the other one, summed in the source domain (Claerbout, 1971, 1985).

The zero-lag cross-correlation imaging condition (ZL-CC-IC) produces spatial low-frequency noise called artifacts due to the superposition between waves such as head, diving and backscattered waves immersed in the source and receiver wavefields and the amplitude of migrated images.

Artifacts reduction has been widely studied and several techniques have been proposed. Youn \& Zhou (2001) used the Laplacian image reconstruction to process each frame from correlation for an individual shot recorded, Fletcher et al. (2005), added a directional damping term to the non-reflecting wave equation proposed by Baysal et al. (1984) and Yoon \& Marfurt (2006) used the Poynting vector to improve the cross-correlation imaging condition.

Kaelin \& Guitton (2006) normalized the image of the crosscorrelation diving by the source or the receiver illumination, Guitton et al. (2007) used the smooth imaging condition and the least square attenuation method, Costa et al. (2009) combined the obliquity factor weight and illumination compensation in the imaging condition, Whitmore \& Crawley (2012) used the inverse scattering theory to attenuate the backscattered waves and Pestana et al. (2014), based on the relation of inversion and imaging, proposed the impedance sensitivity kernel imaging condition combined with Poynting vector.

In this paper, we revisit a recently proposed method to improve the migrated image and diminish the artifacts occurrence by applying a Laguerre Gauss filter with a spiral phase filter to implement a Radial Hilbert transform to process the crosscorrelation images (Paniagua \& Sierra-Sosa, 2016). First, we describe the zero-lag cross-correlation imaging condition (ZL-CCIC) and the Laplacian filtering, which is the regular technique employed to remove the artifacts in RTM images. Second, the proposed method is described in an extended way. Third, we compare the images obtained by zero-lag cross-correlation imaging condition, Laplacian filtering, and the Laguerre Gauss filtering applied to three synthetic datasets, to present evidences from the effectiveness of our imaging implementation to reduce the low-frequency spatial noise. Then, we compare and analyze the Fourier spectra obtained by the 2D spatial fast Fourier transform of the images obtained by zero-lag cross-correlation imaging condition (ZLCC-IC), zero-lag cross-correlation imaging condition plus Laplacian filtering (ZL-CC-IC-LP) and zero-lag cross-correlation imaging condition plus Laguerre-Gauss filtering (ZL-CC-IC-LG) in order to establish quantitative measurements of the spatial spectral features looking for the first step to determine the accuracy and enhancement capability of the method to reach better subsurface structures (Paniagua \& Quintero, 2017a). Finally, by comparison we carefully demonstrate that despite the smoothed velocity models the Laguerre-Gauss filtering preserves well the reflections with their true locations and significantly attenuates the low-frequency noise in the image (Paniagua \& Quintero, 2017b).

\section{THEORETICAL FRAMEWORK}

\section{Zero-Lag Cross-Correlation Imaging Condition}

The zero-lag cross-correlation between the extrapoled source and receiver wavefields is the imaging condition conventionally used in RTM. The zero-lag cross-correlation imaging condition (ZLCC-IC) was proposed originally by Claerbout $(1971,1985)$ and as defined as follows:

$$
I_{c c}(x, z)=\sum_{j=1}^{s_{\max }} \sum_{i=1}^{t_{\max }} S\left(x, z ; t_{i} ; s_{j}\right) R\left(x, z ; t_{i} ; s_{j}\right)
$$

where $z$ and $x$ denote depth and horizontal axis respectively, $t$ is time, $S\left(x, z ; t_{i} ; s_{j}\right)$ is the forward propagated source wavefield, $R\left(x, z ; t_{i} ; s_{j}\right)$ is the backward propagated receiver wavefield, $t_{\max }$ is the total time, $s_{\max }$ is the total number of sources, and $I_{c c}$ is the cross-correlation image.

This imaging condition is kinematically accurate at the reflectors due to the fact that incident and reflected wavefields are coincident in space and time (Liu et al., 2011), but the migrated amplitudes no longer hold any physical meaning. It produces kinematically correct images of the geometry of the subsurface structure (Chattopadhyay \& McMechan, 2008).

Several implementations of RTM using the imaging condition of Eq. (1) have been reported by Baysal et al. (1983, 1984), Youn \& Zhou (2001), Kaelin \& Guitton (2006), among others.

This method can be affected by the backscattered and turning waves in the modeling process, which cause incident and reflected wavefields to be in phase at locations that not correspond to actual reflection points. These wavefields cause strong correlation noise in the seismic image (artifacts) (Whitmore \& Crawley, 2012). These artifacts occur most frequently in shallow parts and 
hard interfaces in the velocity model, and can mask important details in the image (Suh \& Cai, 2009; Jones, 2014).

\section{Laplacian Filtering}

Second-order derivative edge detection techniques are based on spatial second-order differentiation to enhance edges. An edge is marked if a significant spatial change occurs in the second derivative (Pratt, 2001). The edge Laplacian (Laplacian image reconstruction) of an image function $F(x, y)$ is defined as:

$$
G(x, y)=-\nabla^{2}\{F(x, y)\}
$$

where, the Laplacian operator is:

$$
\nabla^{2}=\frac{\partial^{2}}{\partial x^{2}}+\frac{\partial^{2}}{\partial y^{2}}
$$

$G(x, y)$ is zero if $F(x, y)$ is constant or the amplitude changes linearly. If the change rate of $F(x, y)$ is greater than zero $G(x, y)$ exhibits a sign change at the point of inflection in $F(x, y)$. The zero crossing of $G(x, y)$ indicates the presence of an edge. The negative sign from Eq. (2) indicates that the zero crossing of $G(x, y)$ has a positive slope for an edge whose amplitude increases from left to right or bottom to top in an image (Pratt, 2001). Youn \& Zhou (2001) used the Laplacian image reconstruction operator to correlated image frames. The Laplacian image reconstruction is given by:

$$
I_{L P}(x, z)=\frac{\partial^{2}}{\partial x^{2}} I_{c c}(x, z)+\frac{\partial^{2}}{\partial z^{2}} I_{c c}(x, z)
$$

where $I_{c c}(x, z)$ is the image frame registered (zero-lag crosscorrelation image). An effect associated with the application of the Laplacian operator is a $90^{\circ}$ phase shift and an amplitude change due to the second-order differential.

\section{Laguerre-Gauss Filtering}

Linear integral transforms have been used in multiple knowledge fields such as quantum mechanics, quantum theory fields, quantum dispersion, viscoelasticity, circuit theory, dielectric theory, magnetic resonance, optical metrology, among others (Macdonald \& Brachman, 1956; Sierra-Sosa et al., 2013; Sierra-Sosa, 2014; Angel-Toro et al., 2013). In general, these transforms turn a given function $f(z)$ where the $z$ variable may be complex or real valued, into another function $g(\omega)$ where the variable $w$ also may be complex or real valued, these transforms can be defined as:

$$
g(\omega)=\int_{C_{n}} K_{n}(\omega, z) f(z) d z
$$

where $K_{n}$ with $n=1,2$ are generic functions from the variables $z$ and $\omega$ known as transform kernel, for both the transform with $n=1$ as for its inverse with $n=2 ; C_{n}$ denotes the path in the complex plane. In particular, if $f(z)$ is well-known and $K_{1}$ and $C_{1}$ are specified, it is possible to obtain $g(\omega)$ if the function is integrable. On the other hand, this equation is also a linear integral transform for $f(z)$ when $g(\omega), K_{2}$ and $C_{2}$ are specified. For each integral transform, there exists a relation that turns the transformed function into the original function, usually this relation is also an integral transform, which may be written in terms of algebraic operations. There exists a biunivocal relation among the function and its transform (Debnath \& Bhatta, 2010).

If kernels $K_{1}$ and $K_{2}$ are equal and the integration paths between the transform and its inverse are equal, the relation between $f(z)$ and $g(\omega)$ are reciprocal and known as conjugated functions. When these functions are identical except for the sign, like in Hilbert transform, the relations are contra-reciprocal. The integral transforms are useful to obtain a complex distribution similar to the phase from real valued functions, these distributions are known as pseudo-phase maps (Freund \& Freilikher, 1997). Allowing to obtain an analytic signal from a real valued function, analytic signal concept was introduced by Gabor in communications theory for 1-dimensional signals (Debnath \& Bhatta, 2010). Usually, an analytic signal is defined by the difference of the real part of the original signal and its mean value, and an imaginary part obtained by using an integral transform from real signal.

Laguerre-Gauss transform kernel uses a pure-phase function with a vortex structure in frequency domain, defined as $\beta(f x, f y)=\arctan (f y / f x)$. The particular property from this spiral phase function is that is composed by a heavy-side function with a $\pi$ gap when crossing the origin in every angular direction. In the amplitude, the kernel includes a toroidal geometry doing a bandpass filtering depicted in Figure 1.

Let $I(x, y)$ be the original intensity distribution from an image and $\hat{I}\left(f_{x}, f_{y}\right)$ its Fourier transform. $I(x, y)$ may be related with its respective analytic signal $\tilde{I}(x, y)$ by using a transformation kernel $L G\left(f_{x}, f_{y}\right)$

$$
\begin{gathered}
\tilde{I}(x, y)= \\
\iint_{-\infty}^{\infty} L G\left(f_{x}, f_{y}\right) \hat{I}\left(f_{x}, f_{y}\right) e^{2 \pi i\left(f_{x} x+f_{y} y\right)} d f_{x} d f_{y} \\
L G\left(f_{x}, f_{y}\right)= \\
\left(f_{x}+i f_{y}\right) e^{-\left(\frac{f_{x}^{2}+f_{y}^{2}}{\omega^{2}}\right)}=\rho e^{-\left(\frac{\rho^{2}}{\omega^{2}}\right)} e^{i \beta} \\
\text { where } \rho=\sqrt{f_{x}^{2}+f_{y}^{2}} \text { and } \beta(f x, f y)=\arctan (f y / f x)
\end{gathered}
$$




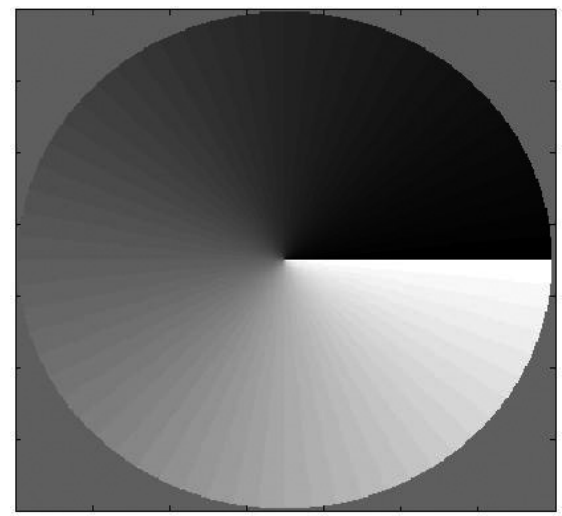

(a)

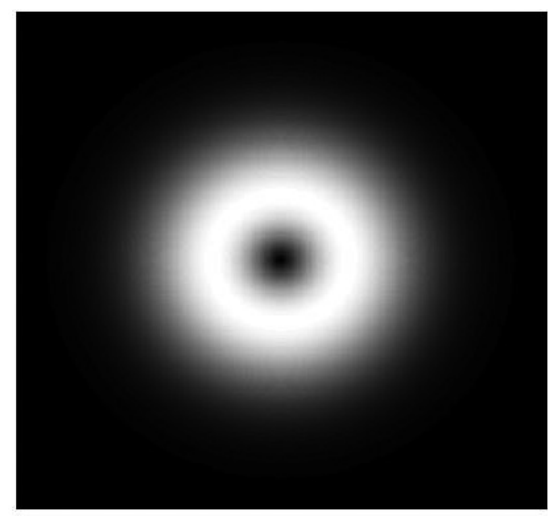

(b)

Figure 1 - (a) Spiral Phase Function; (b) Toroidal amplitude.

are the polar coordinates in the spatial frequency domain. The bandpass filter can be controlled by choosing the bandwidth parameter $\omega$ (Wang et al., 2006), which changes the spatial frequency distribution in the obtained complex field. In this case, $\omega$ tends to one in order to preserve image spatial frequency distribution and perform the bandpass filter component from Laguerre-Gauss.

The Laguerre-Gauss filter allows to realize anisotropic radial Hilbert transform without resolution loss (Gou et al., 2006), and excluding any DC component of the original input function.

\section{METHODOLOGY}

We present a set of steps to develop properly the revisited version of the developed technique relating the preliminary results with the subsurface velocity gradients commonly related to attenuation/impedance properties of the subsurface. We also proposed certain quantitative methods to provide the user a reference point to measure the enhancement of the structural images of the subsurface. Finally, we continue this work by starting the discussion about the capability of the method to perform in a robust way against uncertainties of modeling. The mentioned methodological steps are presented as follows:

1. To apply the Laguerre-Gauss filtering on ZL-CC image and qualitative analysis in simple and complex models and close-up analysis.

2. Establishment of comparative analysis with velocity field gradient to determine the enhancement capability.

3. Quantitative analysis through spatial spectral features extraction (Sierra-Sosa et al, 2016) to characterize the results.
4. Robustness/Uncertainty test with noise contaminated velocity models simulating real uncertainties for RTM.

\section{NUMERICAL EXAMPLES}

We show the results for three synthetic datasets: Two-layer model, 2D SEG-EAGE salt model and 2D Sigsbee 2A model applying the Laguerre-Gauss filter introduced above. We use a reverse time migration (RTM) algorithm with a second- and eighth-order finite difference scheme in time and space, respectively. The RTM images have been obtained using the zero-lag cross-correlation imaging condition, the Laplacian filtering and the Laguerre-Gauss filtering.

\section{Two-layer model}

Consider a synthetic two-layer model with a horizontal and vertical distance of $1.5 \mathrm{~km}$ as shown in Figure 2. We used 21 shot points. The first source is located at $0.25 \mathrm{~km}$ and the last one at $1.25 \mathrm{~km}$ from the beginning of the model; the source interval is $25 \mathrm{~m}$; each shot contains 301 receivers and the receiver interval distance is $5 \mathrm{~m}$.

Figure $2 \mathrm{~b}$ corresponds to the RTM image obtained by crosscorrelation imaging condition (ZL-CC-IC), it can be seen that image is contaminated with low-frequency artifacts (dark shadows) above and near the reflective event and in the shallow parts. In Figure 2c we show the RTM result by using the Laplacian filtering (ZL-CC-IC-LP). The artifacts are reduced, but some highfrequency noise remains in the image close to the surface. On the other hand, the result obtained by applying the Laguerre-Gauss filtering (ZL-CC-IC-LG) is shown in Figure 2d. The artifacts in shallow parts and near the reflective event are significantly reduced and the subsurface structure focalized by the gather shots is more defined and enhanced (left and right spaces are due to the absorbent boundaries). 


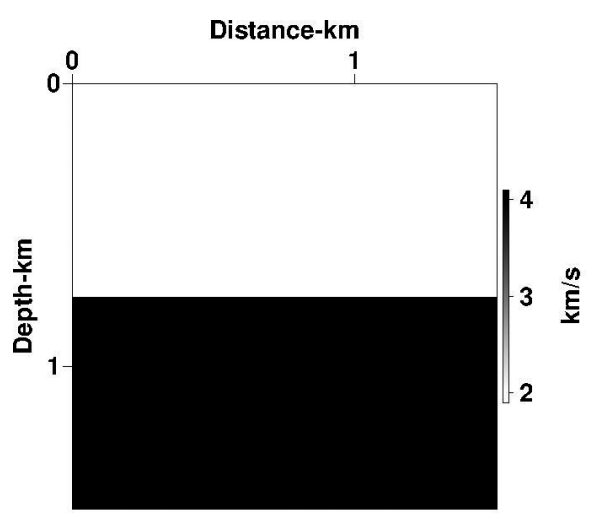

(a)

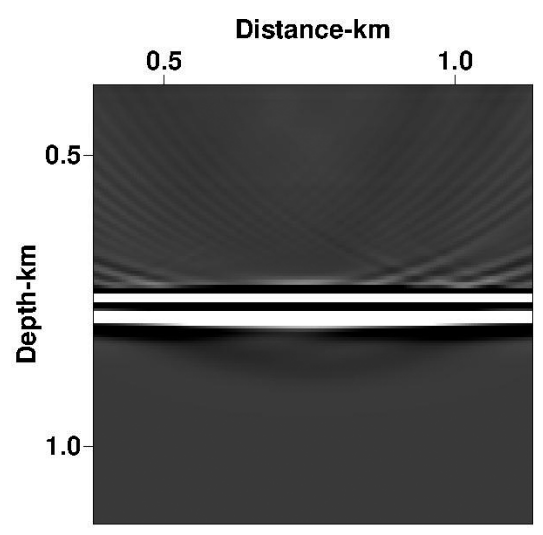

(c)

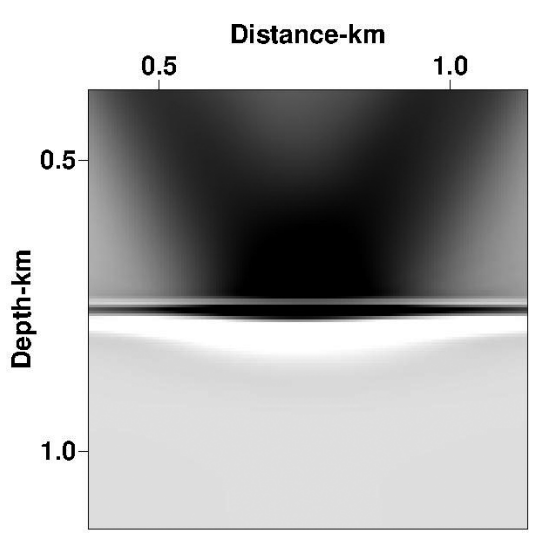

(b)

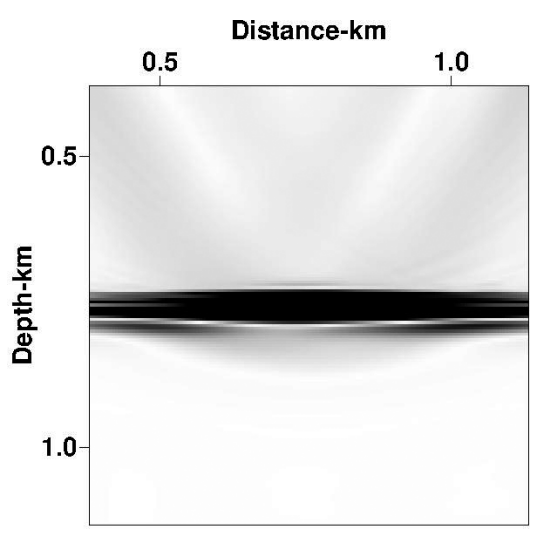

(d)

Figure 2 - Two-layer model RTM using: (a) Velocity model; (b) ZL-CC-IC; (c) ZL-CC-LP; (d) ZL-CC-LG.

An effect associated with the application of the LaguerreGauss filtering is the phase and amplitude changes in the final image. Figure 3 shows the close-up view of the phase of the pseudo complex field (left) corresponding to the image obtained by ZLCC-IC-LG around to the reflector and a vertical line profile (right) at distance $x=0.75 \mathrm{~km}$. We can note that the Laguerre-Gauss filtering changes the reflector phase in $\pi$.

Changes in amplitude are associated with the topological characteristics of the pseudo complex field and will be analyzed in future works to find its relation with seismic attributes such as amplitude and reflectivity.

\section{D SEG-EAGE salt modeI}

To show the major effects of the Laguerre-Gauss filtering, we apply the same strategy to the 2D SEG-EAGE salt model. The length of the model is $4.91 \mathrm{~km}$, the depth of model is $1.14 \mathrm{~km}$. we lay out 32 shot points. The first source is located at $0.19 \mathrm{~km}$ and the last one at $4.72 \mathrm{~km}$. Each shot contains 1290 receivers and the receiver interval distance is $3.81 \mathrm{~m}$.
The 2D SEG-EAGE velocity model is shown in Figure 4a. In Figure 4b the RTM image obtained from zero-lag crosscorrelation imaging condition given by Eq. (1) is shown.

The image is contaminated with artifacts in the shallow parts and near the salt body, and white spots on the bottom caused by backscattering hiding some details of subsurface structures. The artifacts are stronger in the presence of abrupt velocity changes, which are evident near the flanks of the salt body (Jones \& Davison, 2014).

In Figure 4c we show the RTM result using the Laplacian filtering which shows a good reduction of artifacts. To apply this filter reduces low-frequency information and increases the highfrequency noise (Guitton et al., 2007).

Figure $4 \mathrm{~d}$ presents the image from using the Laguerre-Gauss filtering presented in Eqs. (6) and (7). We demonstrate that the Laguerre-Gauss filter removes the undesired low-frequency noise in the RTM images.

Applying the Laguerre-Gauss Filter to cross-correlation image the resulting image is improved: subsurface structures are 

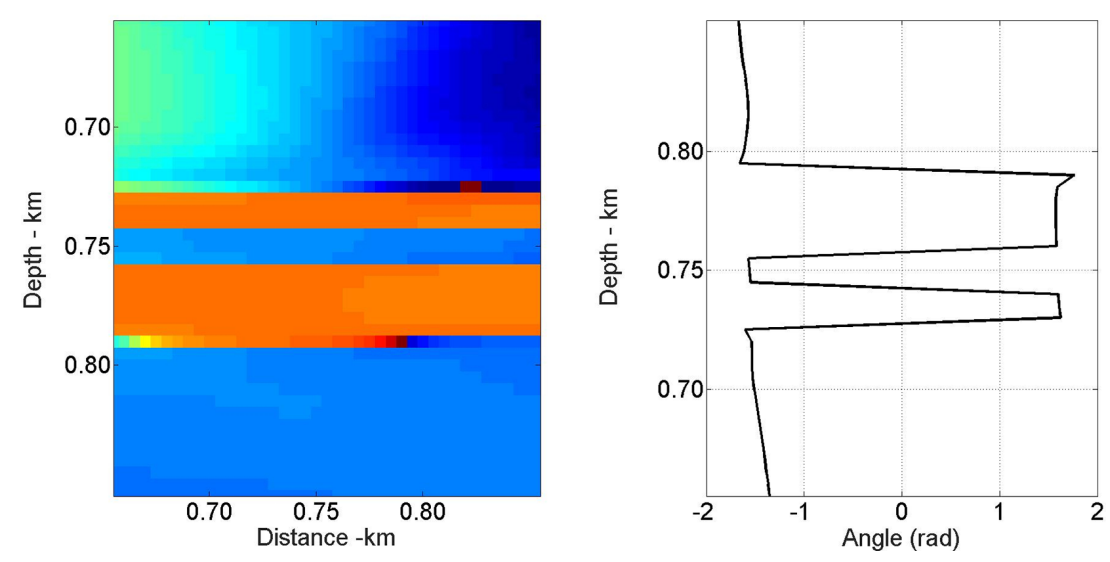

Figure 3 - Phase (left) and vertical line profile (right) of pseudo complex field of Laguerre-Gauss image.

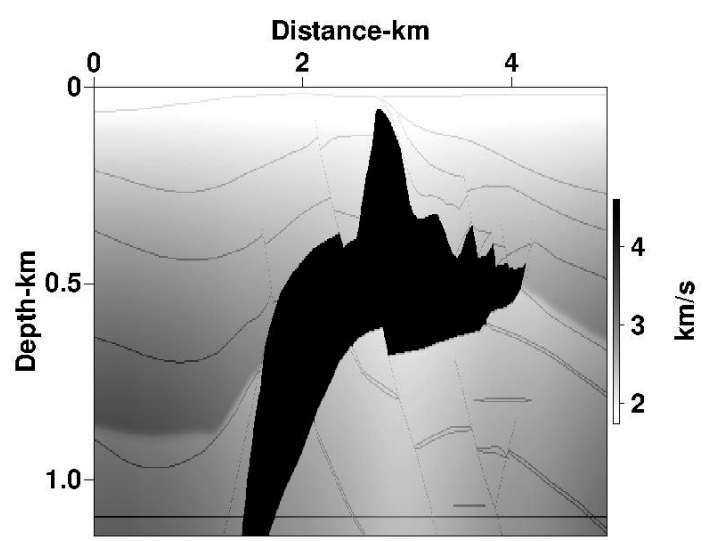

(a)

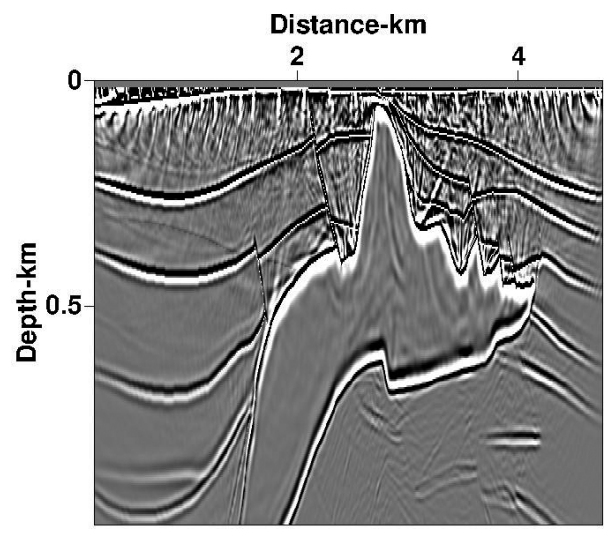

(C)

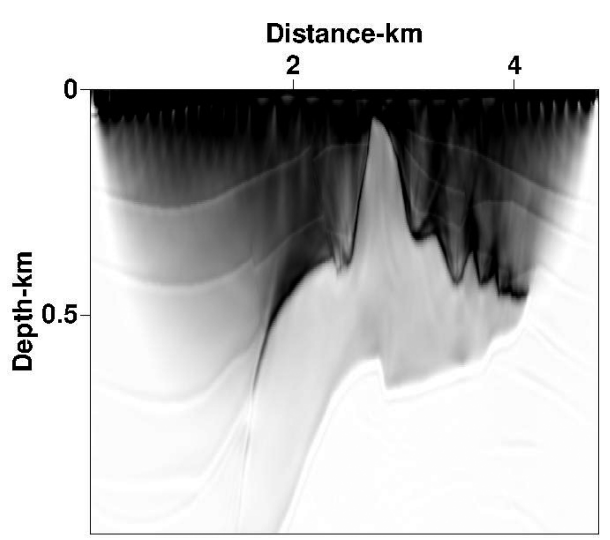

(b)

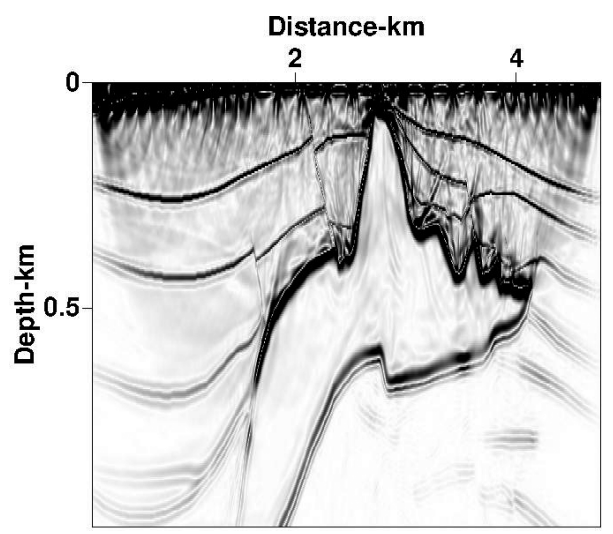

(d)

Figure 4 - 2D SEG-EAGE salt model: (a) Velocity model; RTM result with: (b) Cross-correlation imaging condition; (c) Cross-correlation imaging condition plus Laplacian filter; (d) Cross-correlation imaging condition plus Laguerre-Gauss filter. 


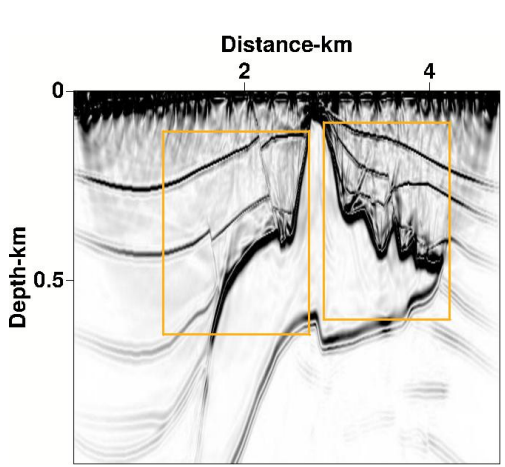

(a)

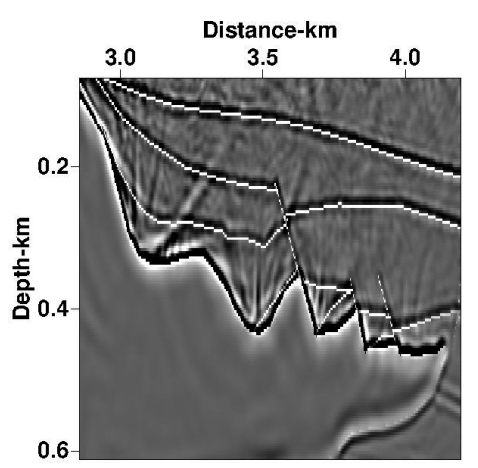

(b)

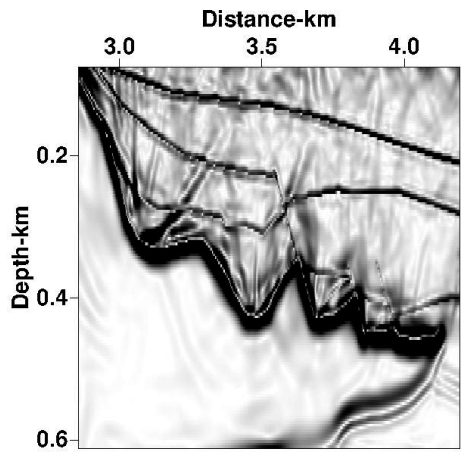

(c)

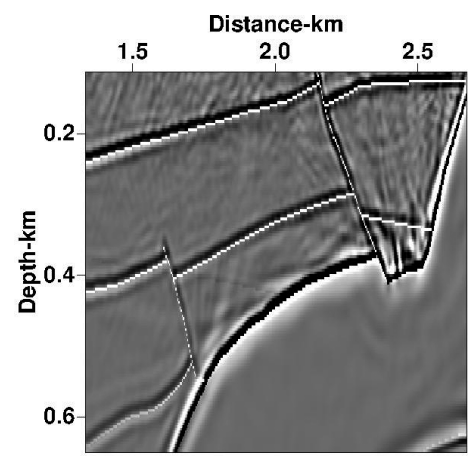

(d)

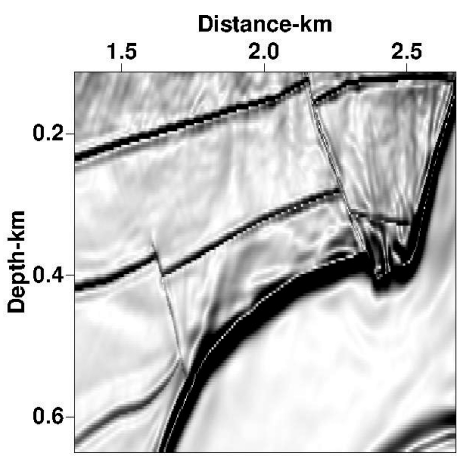

(e)

Figure 5 - Detail of 2D SEG-EAGE RTM image with: (a) Details of the outline regions; (b) and (d) Laplacian filtering; (c) and (e) Laguerre-Gauss filtering.

more defined and the edges of the salt dome are enhanced. The artifacts in shallow parts and near the top salt dome are significantly reduced.

In Figure 5, a close-up view of two sections (marked with yellow boxes) of the migrated image is presented. These were chosen due to its structure complexity. The artifacts reduction in images becomes apparent, by conducting the proposed technique images are improved and the salt body flanks are enhanced.

\section{D Sigsbee 2A model}

Similarly, we apply the Laguerre-Gauss filtering to 2D Sigsbee $2 \mathrm{~A}$ model. Sigsbee $2 \mathrm{~A}$ is a synthetic model of deep water in the Gulf of Mexico. It is characterized by the complex salt shape with rugose salt top found in this area. The velocity model is shown in Figure 6.

In Figure 7 is shown a comparison of images obtained by zero-lag cross-correlation imaging condition, Laplacian and Laguerre-Gauss filtering. The RTM image was obtained using only sixteen shots distributed along the surface. The first source is $10-$ cated at $0.15 \mathrm{~km}$ and the last one at $7.68 \mathrm{~km}$ from the beginning of the model, the source interval is $506.1 \mathrm{~m}$, each shot contains 2133 receivers and the receiver interval distance is $3.75 \mathrm{~m}$.
Figure 7a shows the conventional cross-correlation image where we can be observed that the low-frequency noise is very strong near the salt body. Applying the Laplacian filtering, the low-frequency noise is reduced significantly. This effect is presented in Figure 7b. The result obtained by Laguerre-Gauss filtering is shown in Figure 7c. The noise is strongly reduced near the salt body and the structures are enhanced. Likewise, the effect of sources in the surface are significantly attenuated.

In order to verify that the results obtained by using the proposed technique match the velocity model interfaces, we proceed to overlap the found interface structures using red lines. Red lines are obtained eroding the contrast inverted LaguerreGauss grayscale images, by means of the convolution between the image and a disk with 2-pixel radius. The objects smaller than the mask disappear and a zero is assigned in its place, the positions of the remaining objects are assigned a one. To these binarized image a skeletonization process is applied by obtaining the distance transform of the image. The image skeleton lies along the transform singularities.

Figure 8a presents the interfaces obtained when using the proposed technique overlapped with the SEG-EAGE velocity model, it should be noted the high correspondence between 


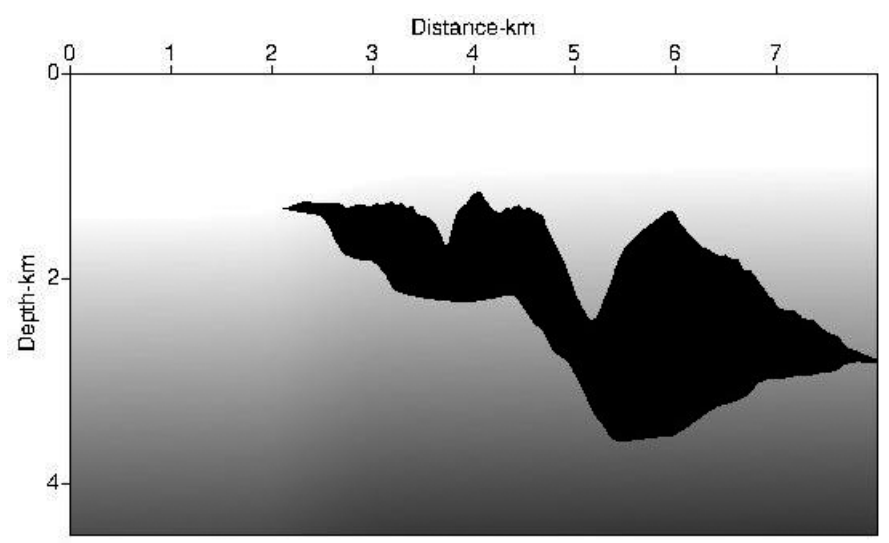

Figure 6 -2D Sigsbee 2A velocity model.

the obtained interfaces with those presented in the model, and Figure $8 \mathrm{~b}$ shows the magnitude of the velocity gradient of the SEG-EAGE velocity model.

We can note that the enhanced edges and flanks of the salt body coincide with the structures present in the velocity model and they are in their true locations. In addition, some details below the salt body are enhanced and better illuminated. The geometry of the salt bodies gives rises to poorly illuminated regions, specifically, below them and the Laguerre-Gauss filter can detect and enhance small amplitude changes. This effect is evident when comparing the image obtained by Cross-correlation imaging condition plus Laguerre-Gauss filter with the magnitude of the velocity gradient in areas below the salt body. Despite of the small changes in the magnitude of the velocity gradient, the structures are enhanced.

\section{EFFECTS OF THE LAGUERRE-GAUSS FILTER IN THE MAGNITUDE SPECTRUM}

In order to describe properly the L-G filter, the magnitude of the spatial Fourier spectrum of the images of both models presented above is shown in Figures 9 and 10 (left column). It is interesting to find out a way to properly compare the results of the post processing procedure so we perform the line profile of the Fourier Spectrum in order to extract features (Sierra-Sosa et al., 2016) and compare the intensity profiles and related them to the enhancement of the edges in the structural image.

The horizontal ( $x$-axis) and vertical $(z$-axis) line profile is evaluated in each spectrum (center and right column, respectively). The top row corresponds to the zero-lag cross-correlation image (ZL-CC-IC) crossing the origin of the Fourier spectrum, the center row to the cross-correlation image plus Laplacian filtering (ZL-CC-IC-LP) and the bottom row to the cross-correlation image plus Laguerre-Gauss filtering (ZL-CC-IC-LG).

\section{Two-layer model}

Figure 9 shows the magnitude of the spatial Fourier spectrum (left) and horizontal (center) and vertical (right) line profiles of the images of the two-layer model showed in Figure 2.

From vertical profile, we can note that the Laplacian filtering modifies the intensity of low frequencies abruptly and increases the intensity in high frequencies. In horizontal profile, the intensity of the line profile was increased in all frequency values. These modifications are evidenced in the strong changes in the shape of the line profiles from the Fourier spectrum of the Laplacian image respect to the Fourier spectrum of cross-correlation image (Paniagua \& Quintero, 2017a).

On the other hand, the Laguerre-Gauss filtering shows a good behavior due to its isotropic feature. In addition to the advantage of spatial isotropic common to the Riesz transform stemming from the spiral phase function, the Laguerre-Gauss transform has the favorable characteristics to automatically exclude any DC component of the original input function (Wang et al., 2006).

The intensity at low and high frequencies are distributed smoothly and homogeneously. The shape of the horizontal line profile of Laguerre-Gauss spectrum is similar to the horizontal line profile of the cross-correlation spectrum. In vertical profile the variations in intensity at high- and low-frequencies are attenuated and smoothed.

\section{D SEG-EAGE salt model}

As presented in previous section for the two-layer model, the Fourier spectra and the horizontal and vertical line profiles are analyzed in order to quantify the effect of the Laplacian and Laguerre-Gauss filtering in the Fourier spectrum of the crosscorrelation image of the 2D SEG-EAGE salt model. 


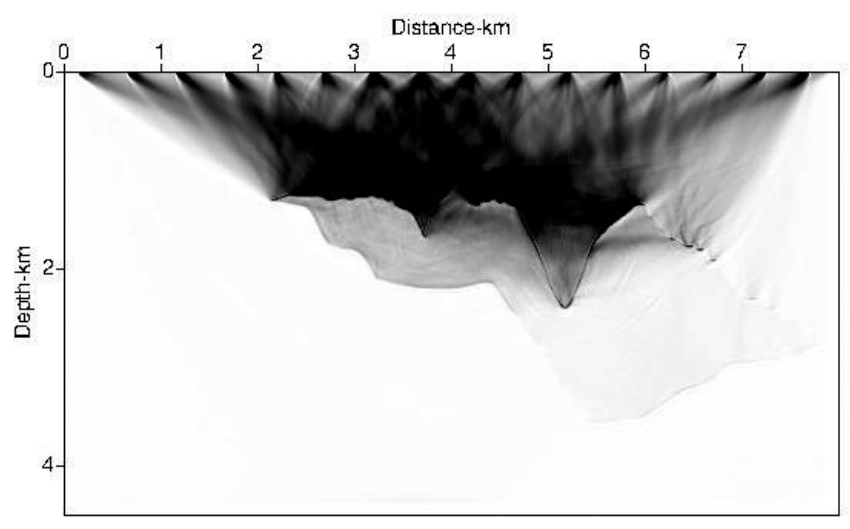

(a)

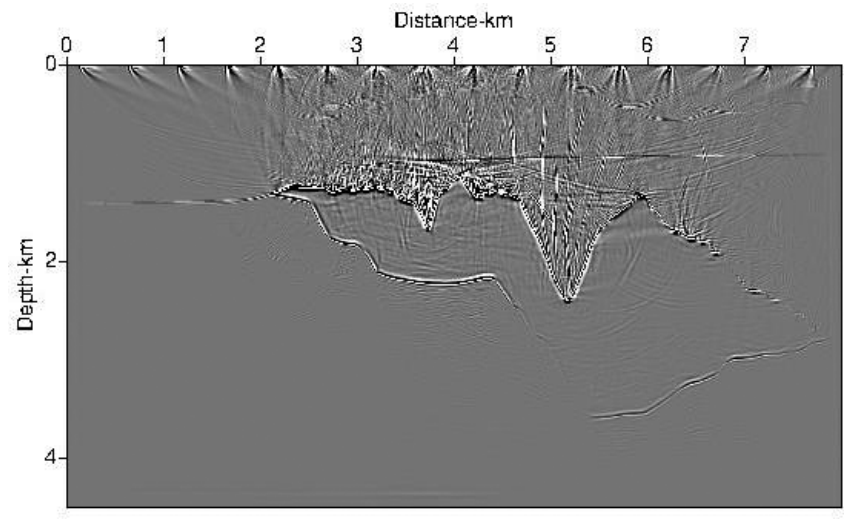

(b)

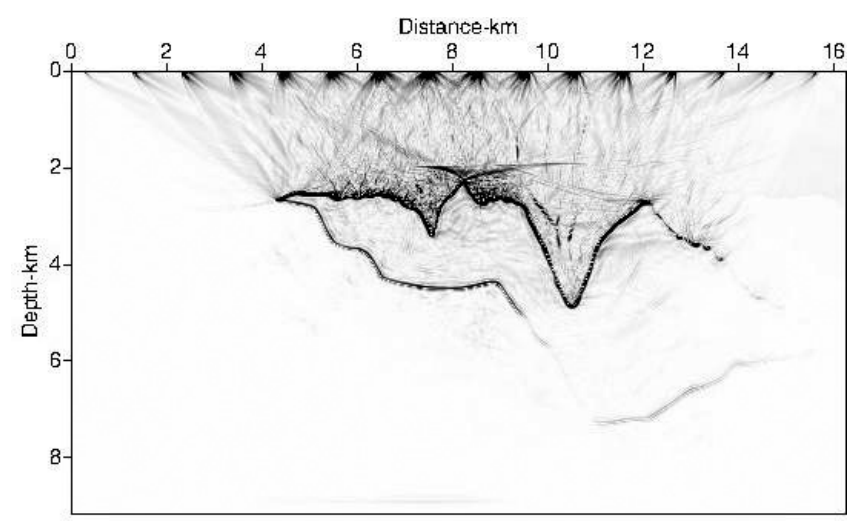

(c)

Figure 7 - 2D Sigsbee 2A RTM using: (a) Cross-correlation imaging condition; (b) Cross-correlation imaging condition plus Laplacian filtering; (c) Cross-correlation imaging condition plus Laguerre-Gauss filtering.

In Figure 10, the top row corresponds to the magnitude spectrum and the horizontal and vertical lines profiles of the cross-correlation image, respectively, the center row to the crosscorrelation image plus Laplacian filtering and the bottom row to the cross-correlation image plus Laguerre-Gauss filtering.
Similarly, horizontal and vertical line profiles cross the origin of the Fourier spectrum.

The Laplacian filtering shows a strong attenuation of the intensity at high- and low-frequency noise, modifying the shape of the line profiles, and changing the intensity in each spatial 


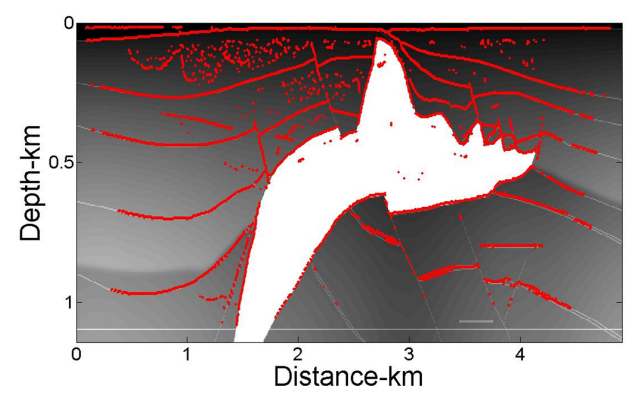

(a)

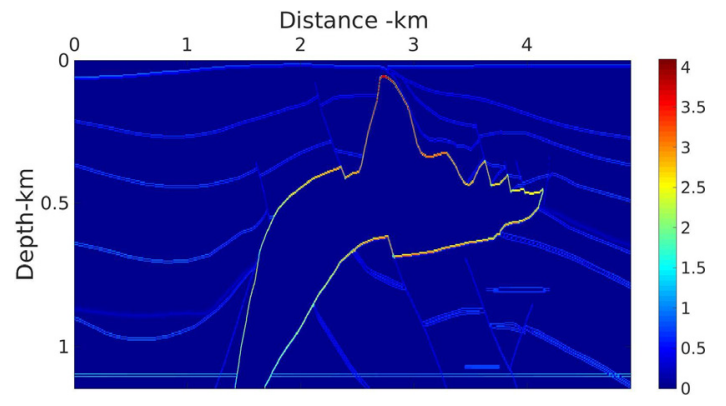

(b)

Figure 8 - (a) Comparison between image obtained with Laguerre-Gauss filtering and the initial model of the 2D SEG-EAGE; (b) Magnitude of the velocity gradient.
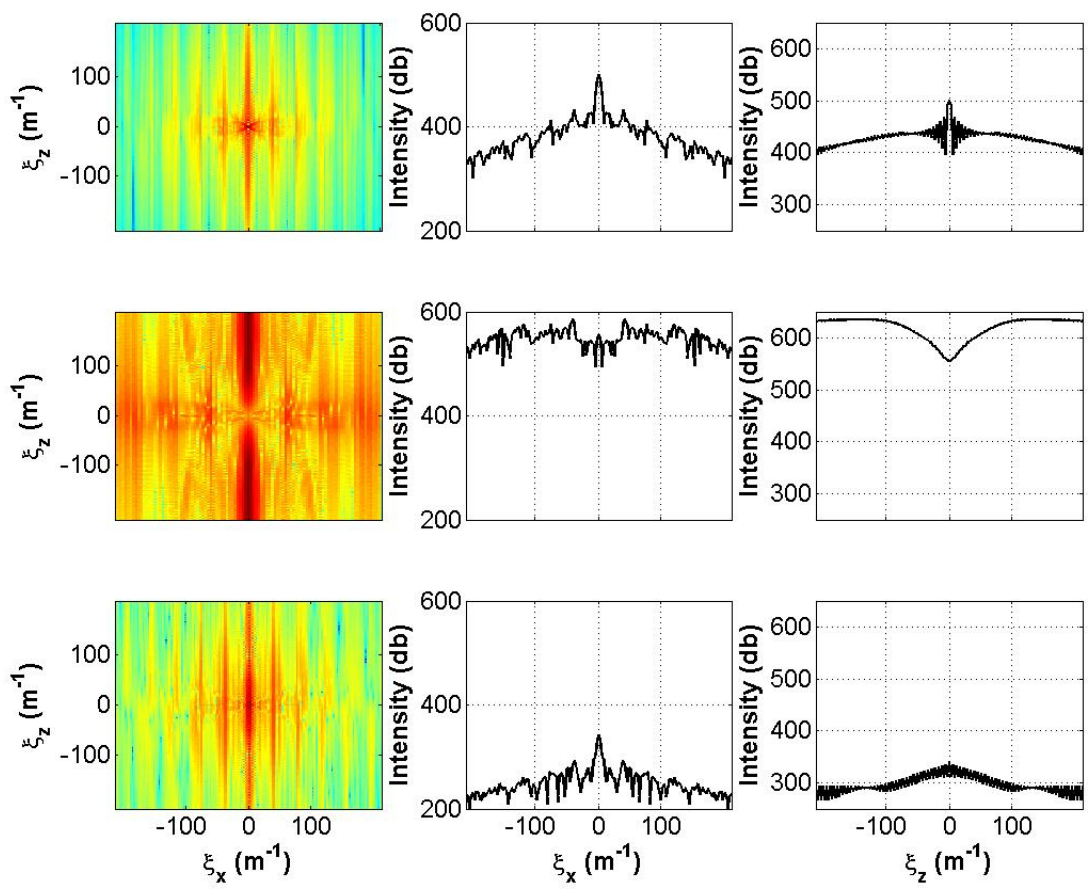

Figure $\mathbf{9}$ - Magnitude of the spatial Fourier spectrum and horizontal and vertical line profiles from images presented in Figure 2.

frequency value. We can note this effect in the shape of the horizontal and vertical line profiles. In the vertical line profile, the reduction is more evident in the intensity at low frequencies values.

Laguerre-Gauss filtering shows that the intensity in all frequency values are modified homogeneously and smoothly. The shape of the horizontal line profile is similar to the shape of the horizontal line profile of the cross-correlation spectrum. From vertical line profile, we can note that the variations of the intensity are smoother and evenly distributed.

\section{SMOOTHED VELOCITY MODELS}

We evaluate the capability of the Laguerre-Gauss filtering on the 2D SEG-EAGE salt model. We applied RTM using the ZL-CC-
IC-LG with the original and two smoothed velocity models. The velocity models was smoothed by using a 2D spatial Gaussian filter with two arbitrary chosen standard deviations of 10 and 20.

The resulting images are shown in Figure 11.

Figure 11b shows the image obtained by ZL-CC-IC-LG using the original velocity model (Fig. 11a). We obtain a good structural image where the artifacts are significantly reduced, the subsurface structures are more defined and the flanks of the salt body are enhanced (Paniagua \& Quintero, 2017b).

In Figures $11 \mathrm{c}$ and $11 \mathrm{~d}$, the velocity model smoothed with the spatial Gaussian filter with standard deviation of 10 and the image obtained by ZL-CC-IC-LG are shown, respectively. The artifacts in shallow parts and near the salt dome are significantly reduced. The edges are enhanced, especially the salt flanks of the 

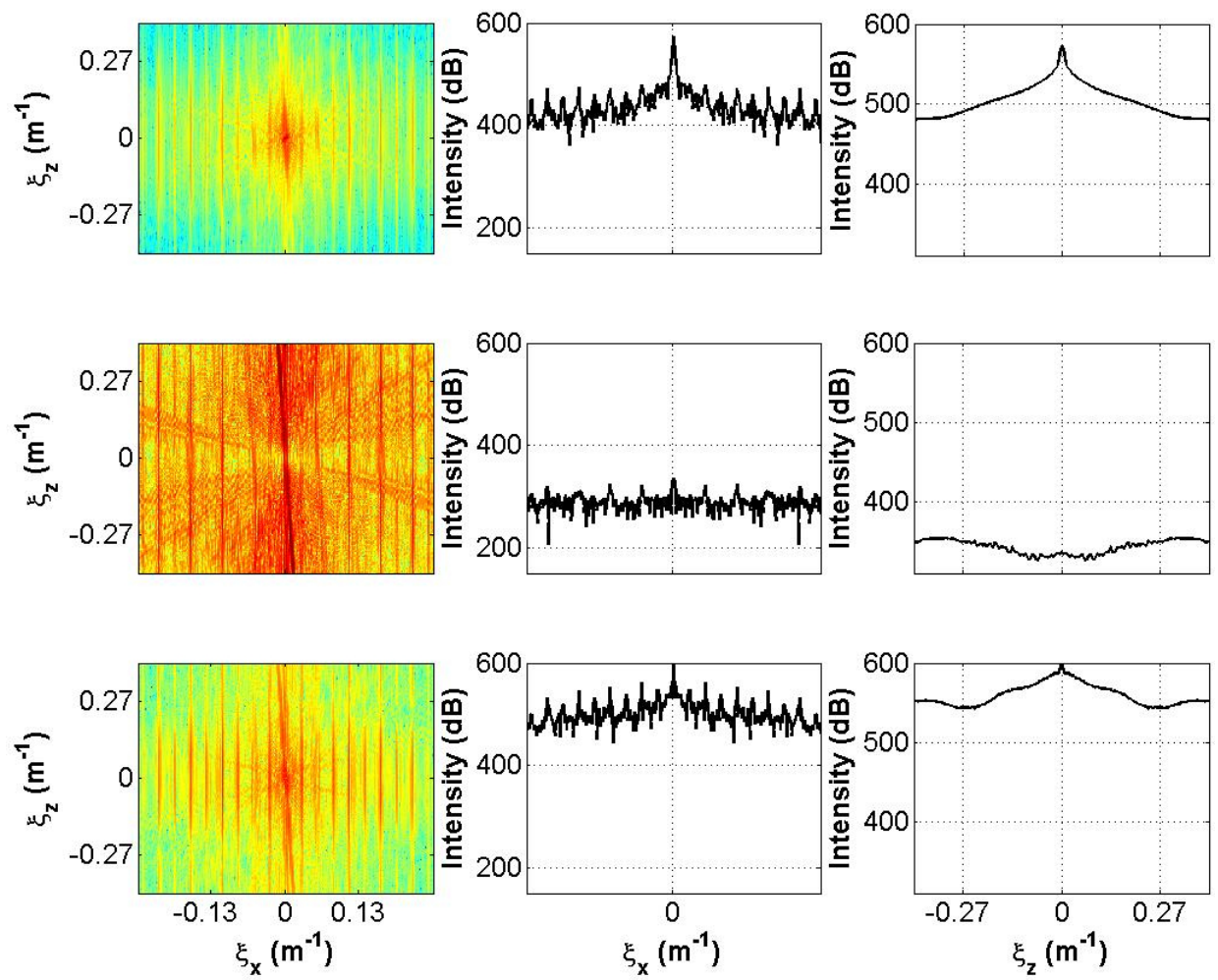

Figure 10 - Magnitude spectra and horizontal and vertical line profiles from images of 2D SEG-EAGE RTM.

salt body. From Figure 11f, we can note that the flanks of the salt body are enhanced, even below the salt dome. Similarly, artifacts in the image are reduced and the subsurface structures are well delineated and located in their correct position. Despite the smoothed velocity model, the Laguerre-Gauss filtering has a good behavior by enhancing the structures and flanks of the salt body and the reduction of low spatial artifacts. It is capable of detecting even very small changes.

\section{CONCLUSIONS}

Zero-lag cross-correlation imaging condition produces spatial low-frequency noise (artifacts) due to the correlation between of the incident and reflected wavefields in points at locations are not reflections points. These artifacts can hide important details in the image. Several techniques have been proposed to remove or attenuated the artifacts and the Laplacian filtering is the commonly used. The effect of the Laplacian filtering is attenuating the lowfrequency noise but it increases the high-frequency noise.

We have proposed a technique to attenuate RTM artifacts after the zero-lag cross-correlation imaging condition. This method uses the Laguerre Gaussian filter to reduce the low- and highfrequency spatial noise and enhances the edges in the image.
We showed that the Laguerre-Gauss filter has a good behavior and distributed homogeneously and smoothly the intensity of the magnitude of the Fourier spectrum due to its isotropic feature. The shape of the line profiles that cross the origin of the Fourier spectrum is similar to the line profiles of the Fourier spectrum of the cross-correlation image but the intensity in all frequency values are distributed smoothly.

In presence of smoothed velocity models, the LaguerreGauss filter obtain similar results, achieving the reduction of lowfrequency noise and preserving the reflective events, enhancing any small change in the seismic image and preserving the true locations of reflections.

To apply this filter to the image obtained with crosscorrelation imaging condition, the low-frequency noise (artifacts) was removed and the reflective events were more defined. This filter enhances the edges without resolution loss.

\section{ACKNOWLEDGMENTS}

We would like to express our gratitude with Colombian Oil Company ECOPETROL and COLCIENCIAS for supporting this work as a part of the research project grant No. 0266-2013. We also are grateful to our families for their support during this research. 


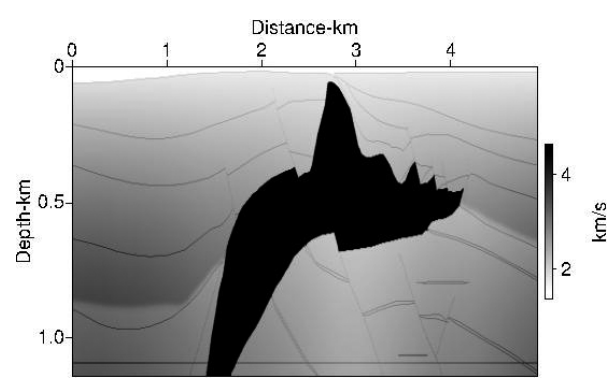

(a)

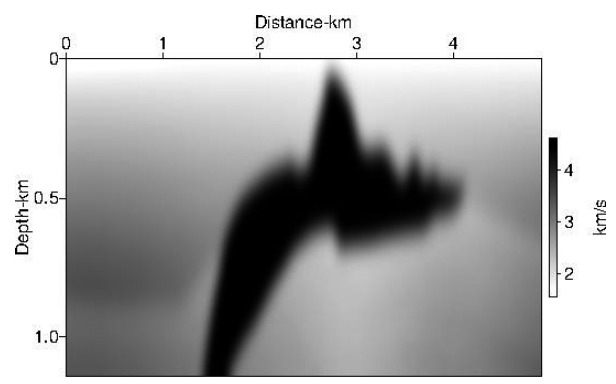

(c)

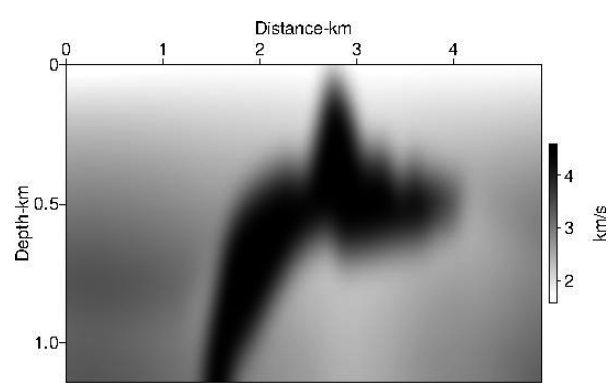

(e)

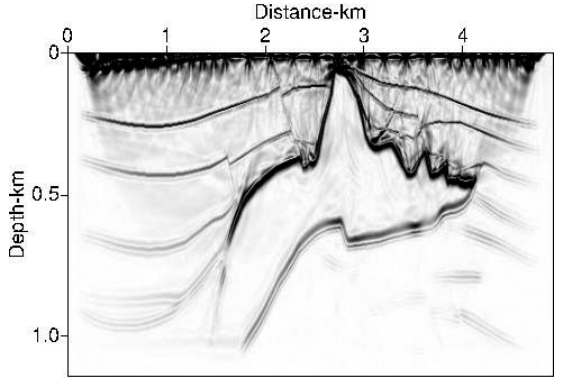

(b)

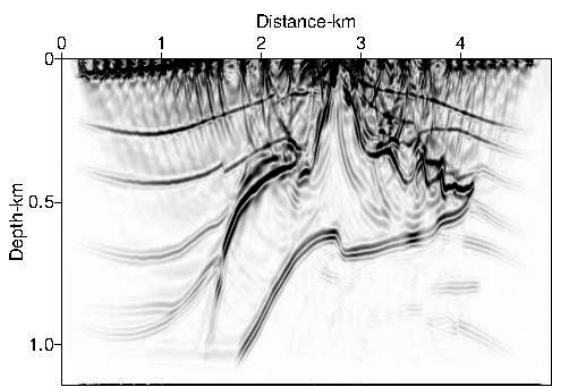

(d)

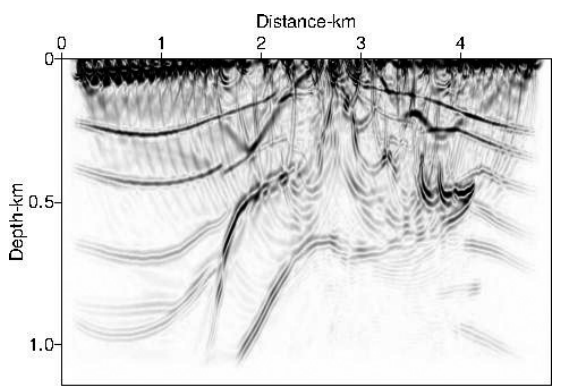

(f)

Figure 11 - The original (a), smoothed with standard deviation of 10 (c) and smoothed with standard deviation of 20 (e) 2D SEG-EAGE model used for migration. RTM results with ZL-CC-IC-LG (b), (d) and (f) for velocity models (a), (c), (e), respectively.

\section{REFERENCES}

ANGEL-TORO L, SIERRA-SOSA D, TEBALDI M \& BOLOGNINI N. 2013. In-plane displacement measurement in vortex metrology. Journal of the Optical Society of America, A, 30(3): 462-469.

BAYSAL E, KOSLOFF DD \& SHERWOOD JW. 1983. Reverse time migration. Geophysics, 48: 1514-1524.

BAYSAL E, KOSLOFF DD \& SHERWOOD JW. 1984. A two-way nonreflecting wave equation. Geophysics, 49: 132-141.

CHATTOPADHYAY S \& McMECHAN GA. 2008. Imaging conditions for prestack reverse-time migration. Geophysics, 73(3): S81-S89.

CLAERBOUT JF. 1971. Toward a unified theory of reflector mapping. Geophysics, 36: 467-481.
CLAERBOUT JF. 1985. Imaging the Earth's interior, Blackwell Scientific Publications, California, $398 \mathrm{pp}$.

COSTA J, SILVA F, ALCÁNTARA R, SCHLEICHER J \& NOVAIS A. 2009. Obliquity-correction imaging condition for reverse time migration. Geophysics, 74: S57-S66.

DEBNATH L \& BHATTA D. 2010. Integral transforms and their applications, CRC press, N.Y., $688 \mathrm{pp}$.

FLETCHER R, FOWLER P \& KITCHENSIDE P. 2005. Suppressing artifacts in prestack reverse time migration. In: 75th International Annual Meeting, SEG, Expanded abstracts: 2049-2051.

FREUND I \& FREILIKHER V. 1997. Parameterization of anisotropic vortices. Journal of the Optical Society of America A, 14: 1902-1910. 
GOU C, HAN Y \& XU J. 2006. Radial Hilbert transform with LaguerreGaussian spatial filters. Optics Letters, 31: 1394-1396.

GUITTON A, KAELIN B \& BIONDI B. 2007. Least-square attenuation of reverse time migration. In: 76th International Annual Meeting, SEG, Expanded abstracts: 2348-2352.

GUITTON A, VALENCIANO A, BEVC D \& CLAERBOUT JF. 2007. Smoothing imaging condition for shot-profile migration. Geophysics, 72: 149-154.

JONES IF. 2014. Tutorial: migration imaging conditions. First Break, 32(12): 45-55.

JONES IF \& DAVISON I. 2014. Seismic imaging in and around salt bodies. Interpretation, 2: SL1-SL20.

KAELIN B \& GUITTON A. 2006. Imaging condition for reverse time migration. In: 76th International Annual Meeting and Exposition, SEG, Expanded abstracts: 2594-2598.

LIU F, ZHANG G, MORTON S \& LEVEILLE J. 2011. An effective imaging condition for reverse time migration using wavefield decomposition. Geophysics, 76: 10-29.

MACDONALD JR \& BRACHMAN MK. 1956. Linear system integral transform relations. Reviews of Modern Physics, 28: 393-422.

PANIAGUA JG \& SIERRA-SOSAD. 2016. Laguerre Gaussian filters in reverse time migration image reconstruction. In: VII Simpósio Brasileiro de Geofísica, SBGf, Expanded abstracts.

PANIAGUA JG \& QUINTERO OL. 2017a. The use of Laguerre-Gauss transform in $2 \mathrm{D}$ reverse time migration imaging. In: 15th International Congress of the Brazilian Geophysical Society, SBGf, Expanded abstracts.

PANIAGUA JG \& QUINTERO OL. 2017b. Attenuation of reverse time migration artifacts using Laguerre-Gauss filtering. In: 79th EAGE Conference and Exhibition. Extended abstracts.
PRATT WK. 2001. Digital image processing, Wiley Interscience, N.Y., $738 \mathrm{pp}$.

PESTANA R, DOS SANTOS A \& ARAUJO E. 2014. RTM imaging condition using impedance sensitivity kernel combined with the Poynting vector. In: SEG Technical Program Expanded Abstracts: 3763-3768.

SIERRA-SOSA D. 2014. Estudio de singularidades de fase con aplicaciones a la metrología. Doctoral dissertation. Facultad de Ciencias Exactas Universidad Nacional de la Plata. 120 pp.

SIERRA-SOSA D, ANGEL-TORO L, BOLOGNINI N \& TEBALDI M. 2013. Novel vortex-transform for high frequency modulated patterns. Optics Express, 21(20): 23706-23711.

SIERRA-SOSA D, BASTIDAS M \& QUINTERO OL. 2016. Double Fourier analysis for emotion identification in voiced speech. Journal of Physics. Conference Series: 705.

SUH SY \& CAI J. 2009. Reverse time migration fan filtering plus wavefield decomposition. In: International Exposition and Annual Meeting, SEG, Expanded abstracts: 2804-2808.

WANG W, YOKOZEKI T, ISHIJIMA R, TAKEDA M \& HANSON SG. 2006. Optical vortex metrology based on the core structures of phase singularities in Laguerre-Gauss transform of a speckle pattern. Optics Express, 14: 10195-10206.

WHITMORE N \& CRAWLEY S. 2012. Applications of RTM inverse scattering imaging conditions. In: 82th Annual International Meeting, SEG, Expanded abstracts: $779-784$.

YOON K \& MARFURT K. 2006. Reverse time migration using the Poynting vector. Exploration Geophysics, 37: 102-107.

YOUN 0 \& ZHOU H. 2001. Depth imaging with multiples. Geophysics, 66: $246-255$.

Recebido em 17 fevereiro, 2017 / Aceito em 16 junho, 2017

Received on February 17, 2017 / Accepted on June 16, 2017 